\title{
Hydrogen bonds of interfacial water in human breast cancer tissue compared to lipid and DNA interfaces
}

\author{
Halina Abramczyk ${ }^{1 *}$, Beata Brozek-Pluska ${ }^{1}$, Jakub Surmacki ${ }^{1}$, Joanna Jablonska-Gajewicz ${ }^{2}$, \\ Radzislaw Kordek ${ }^{2}$ \\ ${ }^{1}$ Laboratory of Laser Molecular Spectroscopy, Institute of Applied Radiation Chemistry, Technical University of Lodz, Lodz, Poland; \\ *Corresponding Author: abramczy@mitr.p.lodz.pl \\ ${ }^{2}$ Medical University of Lodz, Department of Pathology, Chair of Oncology, Lodz, Poland;
}

Received 22 February 2011; revised 17 March 2011; accepted 6 April 2011.

\begin{abstract}
The paper presents the results for water confined in a human breast cancerous tissue, a single stranded DNA, a double stranded DNA and in phospholipids (DPPC-D- $\alpha$-Phosphatidylcholine, dipalmitoyl). The interfacial water in DNA and lipids is represented by a double band in the region of the $\mathrm{OH}$ stretching mode of water corresponding to the symmetric and asymmetric vibrational modes, in contrast to water confined in the cancerous breast tissue where only one band at $3311 \mathrm{~cm}^{-1}$ has been recorded. The marked red-shift of the maximum peak position of the $\mathrm{OH}$ stretching mode confirms that the vibrational properties of the interfacial water observed in restricted biological environment differ drastically from those in bulk water. The change of vibrational pattern of behavior may be due to the decoupling of the vibrations of the $\mathrm{OH}$ bonds in water molecule or change of the vibrational selection rules at biological interfaces. According to our knowledge Raman vibrational properties of water confined in the normal and cancerous breast tissue of the same patient have not been reported in literature yet. Here we have also presented the first Raman 'optical biopsy' images of the non-cancerous and cancerous (infiltrating ductal cancer) human breast tissues.
\end{abstract}

Keywords: H-Bond; Lipids; Breast Cancer; Raman Imaging; Interfacial Water; Vibrational

Spectroscopy

\section{INTRODUCTION}

The properties of water have always been a central subject of investigation. The basic motivation behind such studies is the role of water in biological activity of most molecular processes such as protein-DNA interactions or activity of biological lipid membranes [1-4]. The biological activity depends on stability, structure, and dynamics of water at biological interfaces. The molecular processes in the restricted environments are largely dominated by interactions, vibrational energy transfer, orientation of water molecules, which differ drastically from those of bulk water properties. The confinement of water in biological structures does not represent a single pattern of behavior. For example, water confined in reverse micelles [5-9] differs from water confined in phospholipid membranes [10] or in DNA interfaces [3].

The biological interfaces play an important role in a variety of vital reactions involved in protein interactions, enzyme catalysis, molecular recognition, and various steps of proton and electron transfer pathways. The interactions with water modify static and dynamical properties of lipid bilayers. Moreover, the interactions lead to modification of the diffusion barrier across the membrane to ions and oxygen for example [11]. The modification of the interfaces by water may play an important role in energy dissipation in lipids, which is a key mechanism in maintaining photostability of the biological tissue. Unraveling the role of interfacial water in biological systems has required the application of a number of different techniques. The electron and neutron diffraction, X-ray absorption and diffraction, NMR and electron microscopy have enabled the direct probing of the static structure of hydration patterns. There is a large body of literature that review different aspects of static structure of interfacial water [2,12-17].

Recent developments of time-resolved electron and $\mathrm{X}$-ray methods [18] have enabled the direct probing of the ultrafast structural dynamics. Several reviews have appeared in literature on various aspects of dynamics of interfacial water [1,19-33].

The biological tissue contains both the bulk and the 
confined water. With the advent of clinical imaging (X-ray mammography, magnetic resonance imaging (MRI), ultrasounds (US)) and novel promising methods (near infrared imaging, Raman imaging) valuable tools have been added to extend diagnostic applications such as screening of the breast cancer, which is the most common form of cancer in women. The bulk and the confined water as well as the other significant tissue components (deoxyhemoglobin $(\mathrm{Hb})$, oxyhemoglobin $\left(\mathrm{HbO}_{2}\right)$, adipose) may impede the efficacy of screening in some methods (X-ray mammography, MRI, US). On the other side, information about interfacial water may establish better basis to characterize tissue quantitatively in terms of its optical parameters, such as the scattering and absorption coefficients by optical imaging (Raman, IR, fluorescence) [34,35].

One of the most direct probes to elucidate the structure and dynamics of confined water are the infrared and Raman spectroscopies [1,36-41]. The stationary and time resolved vibrational spectra modified by interaction with biological interfaces are valuable sources of information to extract microscopic picture of the hydration layer. Vibrational properties of the confined water may provide an understanding of interactions with the tissue environment that reflects changes in tissue cellularity, metabolic activity, physiology, and response to cancer. The recent availability of extremely high spatial resolution of optical spectroscopy has led to development of the new techniques such as Raman scanning, which is capable of providing structural information on the nanometer level. As a basic principle of the method is that the specimen is scanned across a laser beam which has a diameter of 200 $\mathrm{nm}$. Measuring the Raman scattering (RS) intensity at each position provides an image of the specimen with a resolution similar to light microscopy. Among the optical methods of diagnostics RS seems to be potentially the most powerful because it provides direct biochemical information from vibrational fingerprint spectra.

In this paper we present our results on vibrational properties of water confined in the non-cancerous and cancerous human breast tissue and compare them with the properties of water confined at the interfaces for selected components of the biological tissue such as DNA (single stranded DNA and a double stranded DNA), phospholipids (DPPC-D- $\alpha$-Phosphatidylcholine, dipalmitoyl). According to our knowledge Raman vibrational properties of water confined in the normal and cancerous breast tissue of the same patient have not been reported in literature yet. The results presented in the paper provide a basis for a substantial revision for interpretation of the origin of the IR and Raman bands observed in the region of the $\mathrm{OH}$ stretching mode of the interfacial water.

\section{EXPERIMENTAL METHODS}

DPPC (D- $\alpha$-Phosphatidylcholine, dipalmitoyl) was purchased from Aldrich (product number: P1652). The double stranded DNA was purchased from Thermo Scientific, Ulm, Germany and was composed of 5'-AAT ATA TAT ATA TAT ATA TAT AA-3' and 5'-TTA TAT ATA TAT ATA TAT ATA TT-3' strands. The single stranded DNA was purchased from Institute of Biochemistry and Biophysics Polish Academy of Science, Warsaw, Poland and had a following sequence: 5'-TTA TAT ATA TAT ATA TAT ATA TT-3'. Scheme 1 presents the systems studied in the paper.

The breast tissue samples were prepared from the material removed in a surgical operation of a patient suffering from an infiltrating ductal breast cancer. The breast tissue samples taken for research did not affect the course of the operation or treatment of the patients. All procedures were conducted under a protocol approved by the institutional Bioethics Committee at the Medical University of Lodz, Poland (RNN/29/11/KE, RNN/30/ $11 / \mathrm{KE}, \mathrm{RNN} / 31 / 11 / \mathrm{KE}$ ). As physiology of the health and diseased tissues are complex and influenced by multiple internal and external factors, we have limited our comparison of the cancerous and non-cancerous samples to the same patient. The total number of patients was 146 [34-35].The histological analysis has been performed by the pathologists from the Medical University of Lodz, Department of Pathology, Chair of Oncology. After general description of the tumor mass, the surgical margins were marked with ink and the material was dissected. The specimens were cut longitudinally into slices about $2 \mathrm{~cm}$ thick and the features of the cross-sections were described. In the next step the appropriate tissue samples from the tumor mass and from the tumor margin, where no carcinoma was detected by the pathologists, were taken for histopathological and Raman analysis. Two types of the breast tissues have been examined by Raman spectroscopy: bulk tissue, and thin sections of $2 \mathrm{~cm}$. The thin sections were obtained by cryosectioning of the bulk tissue with a microtome. The bulk tissue samples require no processing. The samples for histopathological analysis were fixed in $10 \%$ buffered formalin, embedded in paraffin, put on microscopic glasses and stained with hematoxylin and eosin. The adjacent sections of the samples for Raman measurements were not stained. After Raman measurements the thin sections were stained and underwent the histopathological examination.

Our experimental approach employed IR, Raman spectroscopy and Raman imaging. The home made stainless steel cell was constructed to control the water content of the film for IR measurements. The cell was connected to the reservoir where various salt aqueous solutions were placed to maintain the controlled humid- 
ity. The controlled constant humidity has been maintained by compounds in contact with the film within an enclosed space around it. $\mathrm{P}_{2} \mathrm{O}_{5}$, saturated salt aqueous solutions of $\mathrm{CH}_{3} \mathrm{COOK}, \mathrm{NaCl}, \mathrm{NaBrO}_{3}$ and pure water have been used to maintain humidities of $0,23,75,92$, $100 \%$, respectively.

All Raman images and spectra reported here were acquired using a Raman spectrometer Ramanor U1000 (Jobin Yvon) excited with the ion Ar laser (514 nm) and alpha 500 RA (WITec, Ulm, Germany) model. The alpha 500 system consists of an Olympus microscope, coupled with an UHTS spectrometer and a Newton-CCD camera (operating in standard mode with $1024 \times 127$ pixels at $-64^{\circ} \mathrm{C}$ with full vertical binning. The laser beam doubled SHG of the Nd : YAG laser $(532 \mathrm{~nm})$ is focused on the sample with a numerical aperture NA of 0.50 to the laser spot of $200 \mathrm{~nm}$. The average laser excitation power was $10 \mathrm{~mW}$. Before recording the Raman image, the fluorescence in the sample was quenched by illumination with the excitation light during $500 \mathrm{~ms}$ at each point. The quenching of the fluorescence was very effective due to the high optical density provided by the light focusing. IR spectra were recorded using Specord M80, Zeiss. IR-ATR spectra were collected using Nicolet Avatar 330 FT-IR Thermo spectrometer.

The 2D arrays images of ten-thousands of individual Raman spectra were evaluated by the basis analysis method. In this data analysis method each measured spectrum of the 2D spectral array is compared to basis spectra using a least square fit. Such basis spectra are created by from the average spectra from three different areas in the sample. The weight factor in each point is represented as 2D image of the corresponding color and mixed coloring component. The Raman spectra have been analyzed using the principal component analysis (PCA) and MATHLAB least-squares fitting algorithm. The PCA score plots (model-SNV, mean center, first derivative) for all the recorded Raman spectra and all the samples have been obtained [35].

\section{RESULTS}

Our recent papers $[34,35,42]$ on cancer diagnostics by Raman spectroscopy and Raman imaging demonstrate their power as diagnostic tools with the key advantage for breast cancer pathology. The results demonstrate the ability of Raman spectroscopy to accurately characterize cancer tissue and provide evidence that lipids and carotenoids of the tissue may play an essential role as Raman biomarkers that are able to distinguish between normal, malignant and benign types.

The biological tissue represents a very complex system from the chemical, physical, and biological point of view. Each of the basic components of tissue-lipids, fat, collagen, epithelial cell cytoplasm, cell nucleus with complex DNA structure, calcium oxalate dihydrate, calcium hydroxyapatite, $\beta$-carotene, cholesterol, water-represents a complex chemical composition and structure. It is therefore essential to characterize the structure and composition at all levels to understand the complex behavior of such tissues. The detailed Raman spectra analysis of the normal and cancerous breast human tissue has been discussed in our recent papers $[34,35,42]$. This paper concentrates on the vibrational properties of water confined in the non-cancerous and cancerous human breast tissue and compares the results with the properties of water confined at interfaces for the major components of the biological tissue such as DNA (single stranded DNA and a double stranded DNA), and phospholipids (DPPC-D- $\alpha$-Phosphatidylcholine, dipalmitoyl).

Figure 1(a) shows typical Raman spectra of the $\mathrm{OH}$ stretching vibration of water in the normal (non-cancerous), and cancerous bulk breast tissue (infiltrating ductal cancer) compared to bulk neat water. The maximum band positions at $3258 \mathrm{~cm}^{-1}$ and $3410 \mathrm{~cm}^{-1}$ and the band shape in the bulk breast cancerous tissue are identical to those in the neat bulk water.

We could expect that the characteristic features of water molecules near a biological interface, where H-bond network gets locally disrupted, differ significantly from those of bulk due to interactions with lipids, DNA and proteins. Unfortunately, the result from Figure 1(a) indicates simply that there is limited access to the interfacial, confined water by Raman probing in the bulk tissue, which is dominated by the properties of the bulk water.

To check if the way of the sample preparation induces any changes in the Raman features, we have recorded the Raman spectra and images for the thin sections of 2 $\mathrm{cm}$ of the human breast cancer tissue (infiltrating ductal cancer) of the same patient as in Figure 1(a). Detailed inspection into the $\mathrm{OH}$ stretching band (Figure 1(b)) for the thin section of tissue shows markedly different picture-instead of the two bands at $3258 \mathrm{~cm}^{-1}$ and 3410 $\mathrm{cm}^{-1}$ observed in the bulk tissue one can see only an apparently single band with a maximum at $3311 \mathrm{~cm}^{-1}$ which does not reveal any additional bands when analyzed by deconvolution. It indicates that in the thin sections the laser beam is capable to interrogate deeper into hydration layer, which is usually a few (2-3) water molecular diameters thick and Raman scattering becomes sensitive to the characteristic features of water molecules confined near biological interfaces, which differ drastically from those of the bulk water.

To obtain a more illuminating picture of vibrational behavior of water in human breast tissue we have moni- 


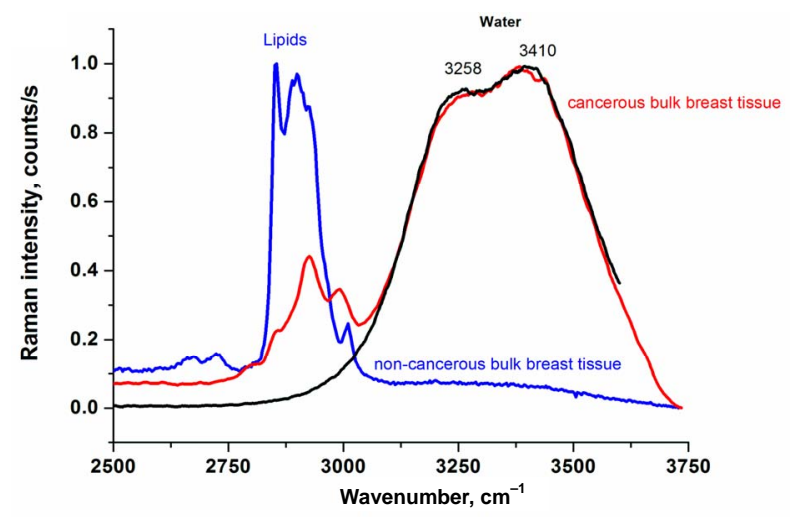

(a)

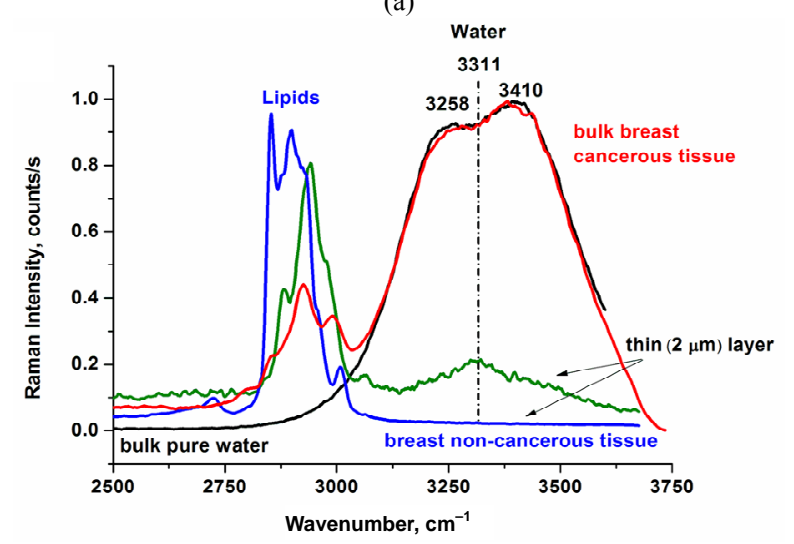

(b)
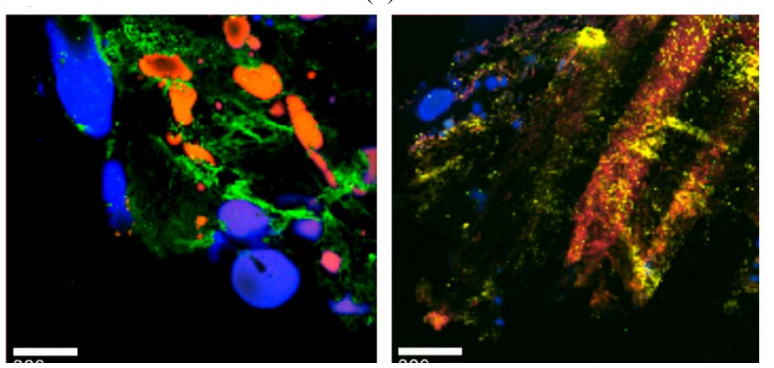

(c)

Figure 1. Raman spectra of the $\mathrm{OH}$ stretching vibration of water in normal (non-cancerous), and cancerous breast tissue (infiltrating ductal cancer) compared to the bulk neat water, (a) bulk tissue; (b) thin layer of $2 \mu \mathrm{m}$ of tissue; (c) Raman images of the normal (non-cancerous)- left and cancerous breast tissue (infiltrating ductal cancer) - right.

tored the Raman spectra in selected constituents of the biological tissue: phospholipids and DNA.

Figure 2 presents the IR spectra of the $\mathrm{OH}$ stretching vibration of water at DPPC surface, which is one of the dominant constituents of epithelium, for various amounts of water. One can see that both the intensities of the bands at $3232 \mathrm{~cm}^{-1}$ and $3379 \mathrm{~cm}^{-1}$ and the maximum peak positions decrease gradually with water content lowering. At the $0 \%$ of humidity the $\mathrm{OH}$ vibrations of the interfacial water have been observed.

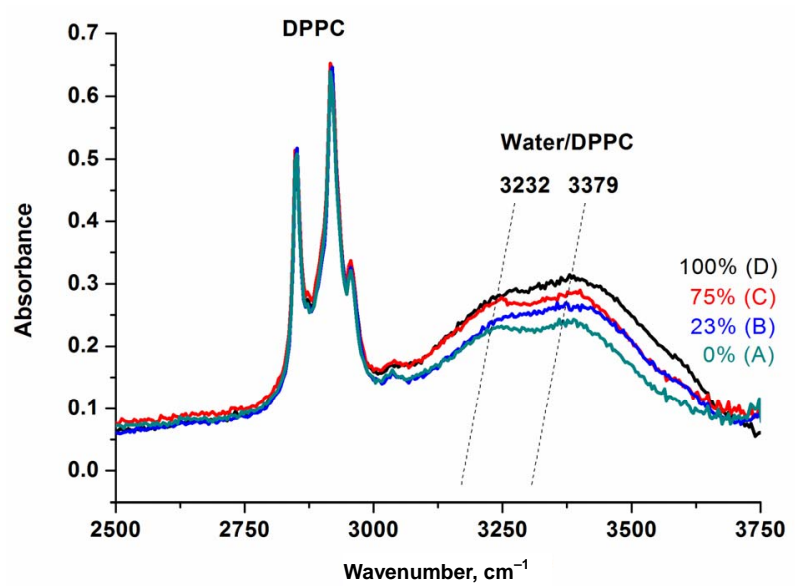

Figure 2. IR spectra of the $\mathrm{OH}$ stretching vibration of DPPC for various content of water (A) $0 \%$, (B) $23 \%$, (C) $75 \%$, (D) $100 \%$.

Figure 3 shows the IR spectra of the $\mathrm{OH}$ stretching vibration of water in a single stranded DNA film, a double stranded DNA film and for comparison at phospholipid interface represented by DPPC. One can see that the interfacial water is very sensitive to the details of biological interfaces. The red shift of the peak positions of the both bands of water is larger in DNA than in phospholipids.

To obtain a better view for discussion, we have summarized the results in Figure 4. Figure 4 shows the spectra of the $\mathrm{OH}$ stretching vibration of water in a single stranded DNA (Figure 4(A)), a double-stranded DNA (Figure 4(B)), DPPC (Figure 4(C)), a thin layer of the cancerous breast tissue (Figure 4(D)), bulk cancerous breast tissue (Figure 4(E)), and bulk water (Figure 4(F)).

Figure 4 demonstrates that in most cases we observe two bands in the region of the $\mathrm{OH}$ stretching vibration of water that are gradually shifted red from bulk water, water at DPPC interface, water at DNA double strand to water at DNA single strand. The only exception is the cancerous breast thin section of the tissue, where only a single band at $3311 \mathrm{~cm}^{-1}$ is observed. The maximum peak positions of the two characteristic peaks are given in Table 1.

\section{DISCUSSION}

A comprehension of the double structure of the bands in the region of the $\mathrm{OH}$ stretching vibration of water and the role of the H-bond network is fundamental to explain the properties of water resulting from different chemical and physical environments. We have studied the human non-cancerous and cancerous breast tissues and prototypical biological systems such as lipids and DNA where different regions are characterized by a specific organi- 


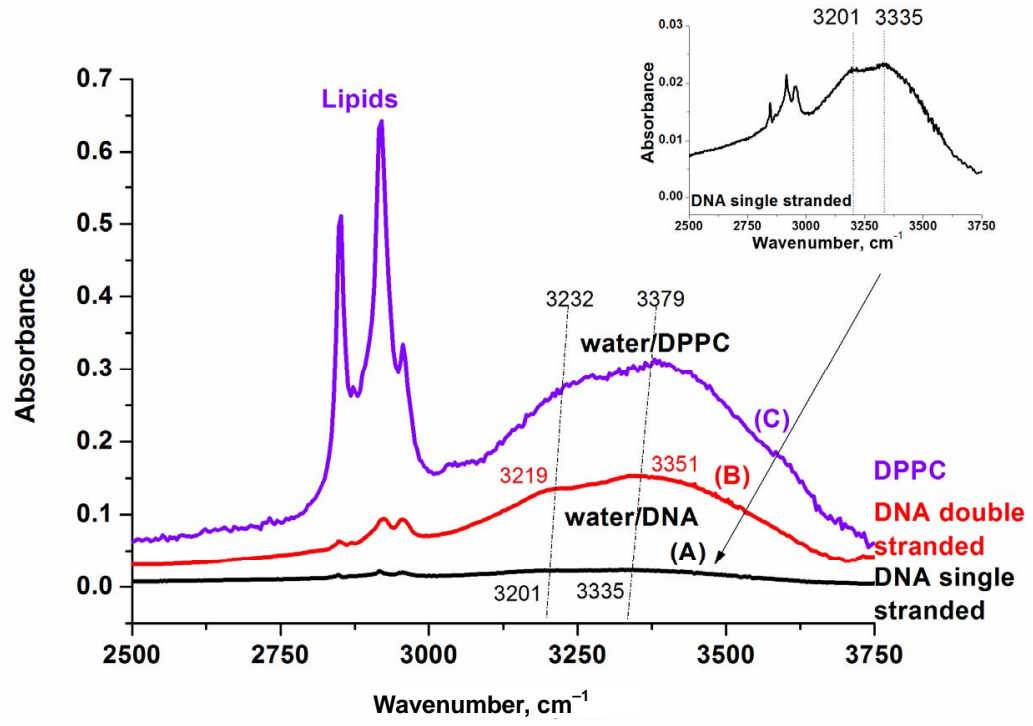

Figure 3. IR spectra of the $\mathrm{OH}$ stretching vibration of the interfacial water in (A) single stranded DNA; (B) double-stranded DNA; (C) DPPC (humidity 0\%).

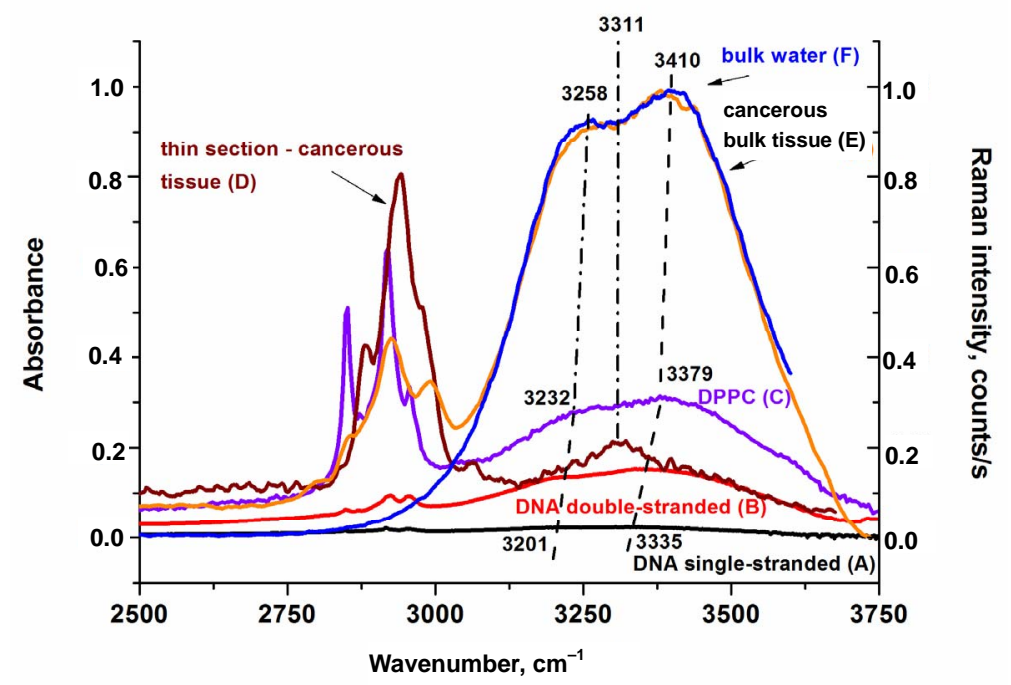

Figure 4. IR spectra of the $\mathrm{OH}$ stretching vibration of water in a single stranded DNA (A); a double-stranded DNA (B); DPPC (humidity 0\%) (C); Raman spectra of the $\mathrm{OH}$ stretching vibration of water in thin section of $2 \mu \mathrm{m}$ of cancerous breast tissue (D); bulk cancerous breast tissue (E); and bulk neat water $(\mathrm{F})$.

Table 1. Maximum peak position of the $\mathrm{OH}$ stretching bands of water at biological interfaces.

\begin{tabular}{ccc}
\hline Sample & $\begin{array}{c}\text { Maximum peak position 1 } \\
{\left[\mathrm{cm}^{-1}\right]}\end{array}$ & Maximum peak position 2 $\left[\mathrm{cm}^{-1}\right]$ \\
\hline DNA- single stranded (IR, ATR) & 3201 & 3335 \\
DNA-duplex (IR, ATR) & 3211 & 3350 \\
DPPC (IR) & 3232 & 3379 \\
Cancerous tissue (bulk) (Raman) & 3258 & 3410 \\
Cancerous tissue (thin layer of 2 $\mu \mathrm{m})$ & & \\
(Raman spectrum) & 3258 & 311 \\
Bulk water (Raman) & & \\
\end{tabular}


zation of the H-bond network.

For an isolated water molecule in the gas phase the double structure in the region of the $\mathrm{OH}$ stretching mode has a clear meaning of the symmetric and the antisymmetric normal modes. In the ideal harmonic approximation there are also no couplings between the intramolecular modes. The bandwidths of the isolated molecule are very narrow, because there are no interactions with the surrounding molecules (bath). In the condensed phase the situation becomes much more complicated. The ideal "symmetric" and "antisymmetric" vibrations, which are decoupled from the other modes and from the environment lose their sense, although vibrations of this sort must obviously still remain. The couplings and bath fluctuations lead to marked bandbroadening, spectacular red shift and complicated band shape structure.

The calculations of the vibrational band shapes of the $\mathrm{OH}$ stretching mode involved in $\mathrm{H}$-bond interactions have a long history and have captured the attention of a number of papers [43-61]. In order to explain spectacular features in frequency shifts, band shapes, and bandwidths of the components the $\mathrm{OH}$ stretching modes the linear and nonlinear response theory [43-45], quantum $\mathrm{ab}$ inito calculations for possible cluster structures [62-68], classical [69-73] and mixed quantum/classical molecular dynamics simulations [74-76] have been used.

The interpretation of vibrational line shapes and shifts at interfaces (particularly at biological interfaces) represents a daunting task, because water in biological systems is represented by relatively large clusters, where different regions are characterized by a specific structure and organization of the H-bond network. Thus, molecules in the interior of the cluster resemble those of the bulk liquid, while molecules at the surface of lipids or DNA have additional specific interactions at the binding sites that change couplings, anharmonicity, symmetry of vibration, and orientational freedom.

Such a mixture of various environments and different time scales leads usually to the situation where neither the inhomogeneous nor adiabatic approximations are appropriate. This statement means that there is a full formal analogy to the vibronic coupling, which originally describes the situation, when the different electronic states are mixed resulting in the coupling between electronic states and vibrational modes. For the vibrational transitions involved in H-bond interactions the term "vibronic coupling" denotes that the motion of low frequency vibrations (X-H..Y stretching and bending vibrations of the $\mathrm{H}$-bond bridge) and high frequency vibrations $(\mathrm{X}-\mathrm{H})$ cannot be separated any longer. As a result the total vibrational wave function cannot be written as a product of the vibrational wave function and the low frequency motion wave function for a given $\mathrm{X}-\mathrm{H}$ vibrational state and more than one vibrational state should be included to describe the total wave function (so called vibrational wave packet).

We have shown that the vibronic coupling is very effective in H-bonded systems [44,45], for an excess electron $[77,78]$ and some proteins $[79,80]$ and determines most of features in static and time resolved spectra including the band width, intensity, band shape and the frequency shift. For H-bonded systems the term 'vibronic coupling' is used to describe the interactions between the high frequency intramolecular vibrational modes $\mathrm{X}-\mathrm{H}$ and the intermolecular low frequency vibrations of the bridge X-H..Y, whereas for the excess electron and proteins 'vibronic coupling' has its original meaning, namely the interactions between the electronic and vibrational modes.

The double structure of the band in the $\mathrm{OH}$ stretch region has been observed for various interfaces: water-air [39], water-lipid [81], water-quartz [82], water-DNA [40]. The detailed discussion of the $\mathrm{OH}$ band shape is beyond the scope of the paper. For the purpose of the presented results we will only mention that literature provides distinct interpretations of the origin of the double band structure of water at the interfaces. The first peak located near $3200 \mathrm{~cm}^{-1}$ has been assigned to the $\mathrm{OH}$ symmetric stretch of the tetrahedrally coordinated water molecules or "ice-like" molecular structure [83,84]. The second peak around $3400 \mathrm{~cm}^{-1}$ has been assigned to water molecules with more disordered hydrogen bonding or "water-like" structure [83]. At water- lipid interfaces the first peak at around $3200 \mathrm{~cm}^{-1}$ has been assigned to the $\mathrm{OH}$ symmetric stretch of water molecules involved in strong interactions with the polar head group of lipids [85], while the second peak at $3400 \mathrm{~cm}^{-1}$ has been assigned to "water-like" structure $[85,86]$. At water-DNA interfaces [40] the first peak around $3200 \mathrm{~cm}^{-1}$ has been assigned to the superposition of the symmetric $\mathrm{NH}_{2}$ stretching mode of adenine and $\mathrm{N}-\mathrm{H}$ stretching mode of thymine, while the second spectral component at 3350 $\mathrm{cm}^{-1}$ to the asymmetric $\mathrm{NH}_{2}$ stretching mode. In contrast to the other referred interpretations the $\mathrm{OH}$ stretching mode of the interfacial water located at the ionic phosphate groups of the DNA backbone has been assigned to the broad band at around $3550 \mathrm{~cm}^{-1}$ [40]. Molecular dynamics simulations [86] assigned the blue-shifted peak to four-coordinated molecules, while the red-shifted peak was attributed to the collective excitations of intermolecular coupled $\mathrm{OH}$ stretching vibrations. Recent molecular dynamics simulations [87] by using a polarizable water model suggested that the red-shifted peak was induced by the intermolecular orientational correlation via anisotropic contribution of the dipole moment. 
One of the recent papers on hydrated phospholipid membranes [81] has assigned the high frequency side (3390 $\mathrm{cm}^{-1}$ ) of the $\mathrm{OH}$ stretching band to weakly H-bonded species in contrast to the low frequency side $\left(3325 \mathrm{~cm}^{-1}\right)$ related almost exclusively to the molecules forming stronger H-bonds with the oxygen atoms of the phosphate or carbonyl groups. The recent paper on DMPC(DL-Dimyristyl-phosphatidylcholine)/water interface [88] has provided a different assignment of the spectral peaks in the $\mathrm{OH}$ stretching region of water. The blue side peak at $3470 \mathrm{~cm}^{-1}$ feature has been attributed to water molecules from the top water layer adjacent to the hydrophilic head group of DMPC. The red side peak at $3290 \mathrm{~cm}^{-1}$ peak has been assigned to the near-bulk water nonadjacent to DMPC. The weak peak at $3590 \mathrm{~cm}^{-1}$ has been attributed to a few water molecules coupled to the glycerol backbone of DMPC.

The comparison of the results for water at biological interfaces is presented in Figure 4. Figure 4 shows that the double structure of bulk water is clearly visible in Raman spectrum with the maxima at 3258 and 3410 $\mathrm{cm}^{-1}$. The double structure occurs also in IR spectrum of water. The IR spectrum of bulk water has also two components [89-91]: at about $3400 \mathrm{~cm}^{-1}$ and a weak shoulder at about $3250 \mathrm{~cm}^{-1}$. The Raman spectrum of the bulk water in Figure 4 represents the spectrum recorded without the polarization analyzer.

The polarized Raman components ( $\mathrm{VV}$ and $\mathrm{VH}$, where the electric vector of the scattered light is parallel and perpendicular to that of the incident light, respectively) give insight into additional features of the band structure of water [93-94].

The parallel (VV) component in Figures 5(a) and 5(b) shows many similarities to the Raman spectrum in Figure 4 in contrast to the perpendicular (VH) component that is more similar to the IR spectrum with a maximum at around $3474 \mathrm{~cm}^{-1}$ and weak shoulders at around 3258 , 3371 and $3598 \mathrm{~cm}^{-1}$. Moreover, the depolarization ratio ( $\rho=\mathrm{I}_{\mathrm{VH}} / \mathrm{I}_{\mathrm{VV}}$ ) as a function of the wavenumber demonstrates almost constant (0.09) magnitude at low wavenumbers, then gradual increase, and again constant value with significantly higher magnitude (0.139) at higher wavenumbers in the spectral range of the $\mathrm{OH}$ stretching modes of water as shown in Figure 5(b). This behavior suggests that the complex band is a superposition of two main bands with different magnitudes of the depolarization ratio, the polarized band $(\rho=0.09)$.. at around $3258 \mathrm{~cm}^{-1}$ and the more depolarized band $(\rho=0.139)$ ataround $3410 \mathrm{~cm}^{-1}$ respectively. The deconvolution of the apparently double structure of the $\mathrm{OH}$ band of water into two components gives two Gaussian bands with maxima at $3208 \mathrm{~cm}^{-1}$ and $3424 \mathrm{~cm}^{-1}$. It indicates that that the idea of symmetric and antisym-

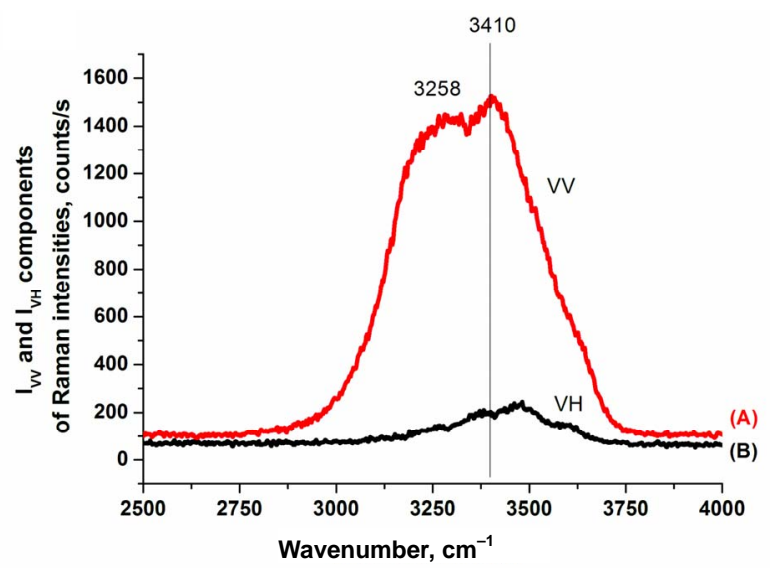

(a)

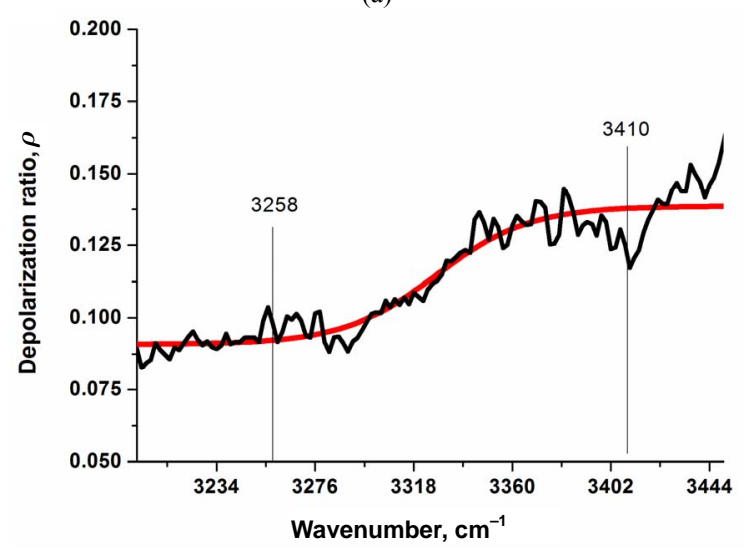

(b)

Figure 5. (a) VV (A) and VH (B) components of Raman spectra of water and depolarization ratio as a function of wavenumber (b).

metric vibrations, that describes perfectly the normal modes of the isolated molecules of water, still operates quite well in the condensed phase. In the view of the results presented so far it is obvious that such vibrations must evidently remain in the condensed phase and the double structure in the region of the stretching mode represents "symmetric" and antisymmetric" like vibrational modes.

Both bands get modified near biological interfaces. One can see from Figure 4 that this indeed happens and the interfacial water is very sensitive to the details of the biological interface. For phospholipid/water and DNA/ water interfaces both $\mathrm{OH}$ stretch components of water are sensitive to the hydrogen-bond interactions in a similar way. One can observe from Figure 4 that the red shift of both bands with respect to the bulk water is 26 $\mathrm{cm}^{-1}$ and $31 \mathrm{~cm}^{-1}$ for the water/lipid interface, respectively. For DNA/water interface the red shift is larger and corresponds to $57 \mathrm{~cm}^{-1}$ and $75 \mathrm{~cm}^{-1}$, respectively. It indicates that the $\mathrm{H}$-bond interactions of water at lipid interface and DNA interface are stronger than those in 
bulk water. The reason for this shift must lie in the fact that water molecules interact with the ionic phosphate groups of the DNA backbone and with the polar head groups of phospholipids (see Scheme 1). The negatively charged phosphate groups in lipids and DNA can form external hydrogen bonds with the surrounding interfacial water molecules. The larger red shift in DNA than in phospholipids may be due to the superposition of the $\mathrm{OH}$ stretch of water with $\mathrm{N}-\mathrm{H}$ modes of adenine and thymine. The larger shift in a single stranded DNA in comparison with a double stranded DNA suggests that water molecules have larger access to form additional H-bonds with thymine and adenine in contrast to the double stranded DNA, where this access is limited (see Scheme 1).

The interpretation of vibrational line shapes and shifts at biological interfaces of the cancerous breast tissue represents much more complicated task. The picture that emerges from Figure 4 shows that the single band at $3311 \mathrm{~cm}^{-1}$ of water at the cancerous breast tissue interface, instead of two $\mathrm{OH}$ bands, is observed in contrast to phospholipids (DPPC)/water and DNA/water interfaces. A characterization of the biological tissue "surface" to which the water molecules are associated is extremely difficult. Fortunately, the analysis of other vibrational bands of the tissue provides some hints. This additional information is demonstrated by the vibrations of fatty acids in the adipose tissue filling the spaces around the lobules and ducts and the fatty acids that make up the cell membrane and nuclear membrane. We have found that the fatty acid composition of the cancerous breast tissue is markedly different from that of the surrounding non-cancerous breast tissue as one can see from the vibrational profiles at around $2800-3000 \mathrm{~cm}^{-1}$ in Figure 1(b). The details will be discussed in our next paper. Here let us briefly conclude by saying that the cancerous breast tissue is dominated by the metabolism products of some fatty acids, while the non-cancerous breast tissue is dominated by monounsaturated oleic acid derivatives. This finding is able to explain the drastic differences observed in Figure 1(b) in the intensities of the $\mathrm{OH}$ bands of water in the non-cancerous and cancerous breast tissues. The non-cancerous tissue shows a negligible signal of water than the cancerous tissue due to higher content of hydrophobic adipose tissue Indeed, the Raman imaging presented in Figure 1(c) shows evidently that the breast structure of the normal tissue contains significantly higher content of the adipose cells (demonstrated in the Raman imaging by the blue color in Figure 1(c), left) than the cancerous tissue (Figure 1(c), right). The amount of water in the thin cancerous tissue of $2 \mathrm{~m}$ is extremely low (as shown by the relative Raman intensities of the $\mathrm{OH}$ stretching bands for the bulk and thin layer tissues in Figure 4). Water in the cancerous breast tissue is very likely present as interfacial, complexed molecules, hydrogen bonded to some hydrophilic moieties.

Further research is needed to establish the origin of the observed vibrational features in this spectral region. First, the idea of decoupled vibration of the $\mathrm{OH}$ bond involved in $\mathrm{H}$-bond interaction to the hydrophilic sites of the tissue has to be tested. Briefly, the idea of the decoupled vibration can be easily explained by the analogy to the deuteration effect. In $\mathrm{HOH}$ molecule there are symmetric and asymmetric vibrations. Upon deuteration the HOD species become decoupled and exhibit the $\mathrm{OH}$ and $\mathrm{OD}$ vibrations in the separate spectral ranges. If one of the $\mathrm{OH}$ bonds in interfacial water molecule interacts strongly with polar (or H-bonded) biological moieties of the tissue the decoupling effect similar to deuteration may occur.

The other explanation may be related to the changed surface selections rules of Raman spectroscopy. The change of the vibrational selections rules at the biological interface may have some analogy to SERS (surface enhanced Raman scattering) and can be due to the perturbations in the optical parameters of the adsorbate (in this case the interfacial water at the biological tissue). The only difference is lack of the contribution from the electromagnetic enhancement mechanism produced by certain rough metal interface in SERS. With this interpretation the band at $3311 \mathrm{~cm}^{-1}$ could be assigned to the $\mathrm{OH}$ asymmetric stretching mode of interfacial water at the tissue surface. The observed shift to red would be the largest one indicating further strengthening of the $\mathrm{H}$-bond of the interfacial water in the human cancerous breast tissue in comparison with the bulk water, the interfacial water at phospholipids membranes and at DNA surfaces.

\section{CONCLUSIONS}

The paper has illustrated important aspects of the complex vibrational features of the $\mathrm{OH}$ water stretching bands in human breast tissue compared to the bulk water, lipids/water and DNA/water interfaces. Here we have also presented the first Raman 'optical biopsy' images of the non-cancerous and cancerous (infiltrating ductal cancer) human breast tissues. The results will help clinicians to accurately assess the diagnostic information contained the Raman spectroscopic and imaging spectroscopies. In our previous papers [34,35] we have demonstrated the role of Raman spectroscopy in diagnosing normal, benign, and malignant human breast tissues with carotenoids and lipid profile as the main biomarkers. Here, we present the results that may suggest that cell hydration is also a factor of particular significance in mechanism of carcinogenesis. From a clinical 
perspective, the abnormally high water content of cancer cells permits the use of Raman technology for tumor detection and treatment.

The essential detailed findings can be summarized as follows:

1) The presented results demonstrate that Raman and IR spectra of the $\mathrm{OH}$ stretching vibrations of water are sensitive indicators of $\mathrm{H}$-bond interactions at various biological interfaces and they differ drastically from vibrational features of bulk water.

2) The $\mathrm{OH}$ stretching vibrations of water can be useful as a potential Raman biomarker to distinguish between the cancerous and non-cancerous human breast tissues. We have found that water amount as well as the band shapes of the $\mathrm{OH}$ stretching bands of the cancerous breast tissue are markedly different from that of the surrounding non-cancerous breast tissue.

3) Water at DNA and lipid interfaces is represented by the bands of symmetric and antisymmetric like vibrations in the region of the $\mathrm{OH}$ stretching modes in contrast to water confined in the cancerous breast tissue where the single band at $3311 \mathrm{~cm}^{-1}$ dominates in the Raman spectrum.

4) Water in the cancerous breast tissue is very likely present as interfacial molecule, which is hydrogen bonded to some hydrophilic moieties in the tissue.

5) The change of vibrational pattern of behavior may be due to the decoupling of the vibrations of the $\mathrm{OH}$ bonds in water molecule or change of the vibrational selection rules at biological interfaces.

\section{ACKNOWLEDGEMENTS}

We gratefully acknowledge the support of this work through the grant 3845/B/T02/2009/37, the grant 4097/B/T02/2010/38 and the Dz. St. 2011.

\section{REFERENCES}

[1] Bagchi, B. (2005) Water Dynamics in the hydration layer around proteins and micelles. Chemical Reviews, 105, 3197-3218. doi:10.1021/cr020661+

[2] Schwabe, J.W.R. (1997) The role of water in protein-DNA interactions. Structural Biology, 7, 126-134.

[3] Pal, S.K., Zhao, L. and Zewail, A.H. (2003) Water at DNA surfaces: Ultrafast dynamics in minor groove recognition. Proceedings of the National Academy of Sciences, 100, 8113-8118. doi:10.1073/pnas.1433066100

[4] Reddy, Ch.K., Dass, A. and Jayaram, B. (2001) Do water molecules mediate protein-dna recognition? Journal of Molecular Biology, 314, 619-632. doi:10.1006/jmbi.2001.5154

[5] Tan, H.S., Piletic, I. R. and Fayer, M.D. (2005) Orientational dynamics of water confined on a nanometer length scale in reverse micelles. Journal of Chemical Physics, 122, Article ID: 174501. doi:10.1063/1.1883605

[6] Tan, H.S., Piletic, I.R., Riter, R.E., Levinger, N.E. and
Fayer, M.D. (2005) Dynamics of water confined on a nanometer length scale in reverse micelles: Ultrafast infrared vibrational echo spectroscopy. Physical Review Letters, 94, Article ID: 057405.

doi:10.1103/PhysRevLett.94.057405

[7] Piletic, I.R., Tan, H.S. and Fayer, M.D. (2005) Dynamics of nanoscopic water: Vibrational echo and infrared pump-probe studies of reverse micelles. Journal of Physical Chemistry B, 109, 21273-21284. doi:10.1021/jp051837p

[8] Piletic, I.R., Moilanen, D.E., Spry, D.B., Levinger, N.E. and Fayer, M.D. (2006) Testing the core/shell model of nanoconfined water in reverse micelles using linear and nonlinear IR spectroscopy. Journal of Physical Chemistry $A$, 110, 4985-4999. doi:10.1021/jp061065c

[9] Dokter, A.M., Woutersen, S. and Bakker, H.J. (2006) Inhomogeneous dynamics in confined water nanodroplets. Proceedings of the National Academy of Sciences, 103, $15355-15358$. doi: $10.1073 /$ pnas.0603239103

[10] Jacobs, R.E. and White, S.H. (1989) The nature of the hydrophobic binding of small peptides at the bilayer interface: Implications for the insertion of transbilayer helices. Biochemistry, 28, 3421-3437. doi:10.1021/bi00434a042

[11] Gruszecki, W.I. and Strzałka, K. (2005) Carotenoids as modulators of lipid membrane physical properties. Biochemica et Biophysica Acta, 1740, 108-115.

[12] Teeter, M.M. (1991) Water-protein interactions: Theory and experiment. Annual Review of Biophysics and Biophysical Chemistry, 20, 577-600. doi:10.1146/annurev.bb.20.060191.003045

[13] Williams, M.A., Goodfellow, J.M. and Thornton, J.M. (1994) Buried waters and internal cavities in monomeric proteins. Protein Science, 3, 1224-1235. doi: $10.1002 /$ pro. 5560030808

[14] Burling, F.T., Weis, W.I., Flaherty, K.M. and Brunger, A.T. (1996) Direct observation of protein solvation and discrete disorder with experimental crystallographic phases. Science, 271, 72-77. doi:10.1126/science.271.5245.72

[15] Berman, H.M. (1994) Hydration of DNA: Take 2. Current Opinion in Structural Biology, 4, 345-350. doi:10.1016/S0959-440X(94)90102-3

[16] Eisenstein, M. and Shakked, Z. (1995) Hydration patterns and intermolecular interactions in A-DNA crystal structures. Implications for DNA recognition. Journal of Molecular Biology, 248, 662-678. doi:10.1006/jmbi.1995.0250

[17] Egli, M., Portmann, S. and Usman, N. (1996) RNA hydration: A detailed look. Biochemistry, 35, 8489-8494. doi:10.1021/bi9607214

[18] Chergui, M. and Zewail, A.H. (2009) Electron and X-ray methods of ultrafast structural dynamics: Advances and applications. ChemPhysChem: A European Journal of Chemical Physics and Physical, 10, 28-43.

[19] Pratt, L.R. and Pohorille, A. (2002) Hydrophobic effects and modeling of biophysical aqueous solution interfaces. Chemical Reviews, 102, 2671-2692. doi:10.1021/cr000692+

[20] Benjamin, I. (1996) Chemical reactions and solvation at liquid interfaces: A microscopic perspective. Chemical 
Reviews, 96, 1449-1476. doi:10.1021/cr950230+

[21] Nandi, N., Bhattacharyya, K. and Bagchi, B. (2000) Dielectric relaxation and salvation dynamics of water in complex chemical and biological systems. Chemical Reviews, 100, 2013-2045. doi:10.1021/cr980127v

[22] Bagchi, B. and Biswas, R. (1998) Ionic mobility and ultrafast solvation: Control of a slow phenomenon by fast dynamics. Accounts of Chemical Research, 31, 181-187. doi:10.1021/ar970226f

[23] Bagchi, B., Oxtoby, D.W. and Fleming, G.R. (1994) Theory of the time development of the Stokes shift in polar media. Chemical Physics, 86, 257-267. doi:10.1016/0301-0104(84)80014-2

[24] Van der Zwan, G. and Hynes, J.T. (1985) Time-dependent fluorescence solvent shifts, dielectric friction, and nonequilibrium solvation in polar solvents. Journal of Physical Chemistry, 89, 4181-4188. doi:10.1021/j100266a008

[25] Friedman, H.L., Reineri, F.O. and Resat, H. (1993) Molecular liquids. In: Teixeira-Diaz, J. Ed., NATO-ASI Series, Kluwer Academic Publishers, Amsterdam.

[26] Stratt, R.M. and Maroncelli, M. (1996) Nonreactive dynamics in solution: The emerging molecular view of solvation dynamics and vibrational relaxation. Journal of Physical Chemistry, 100, 12981-12996. doi:10.1021/jp9608483

[27] Barbara, P.F. and Jarzeba, W. (1990) Ultrafast photochemical intramolecular charge and excited state salvation. Advances in Photochemistry, 15, 1-68. doi:10.1002/9780470133453.ch1

[28] Maroncelli, M. MacInnis, J. and Fleming, G.R. (1989) Polar solvent dynamics and electron-transfer reactions. Science, 243, 1674-1681. doi:10.1126/science.243.4899.1674

[29] Bagchi, B. (1989) Dynamics of solvation and charge transfer reactions in dipolar liquids. Annual Review of Physical Chemistry, 40, 115-141. doi:10.1146/annurev.pc.40.100189.000555

[30] Rossky, P. J. and Simon, J.D. (1994) Dynamics of chemical processes in polar solvents. Nature, 370, 263-269. doi:10.1038/370263a0

[31] Fleming, G.R. and Cho, M. (1996) Chromophore-solvent dynamics. Annual Review of Physical Chemistry, 47, 109-134. doi:10.1021/jp0213506

[32] Pal, S.K., Peon, J., Bagchi, B. and Zewail, A.H. (2002) Biological water: Femtosecond dynamics of macromolecular hydration. Journal of Physical Chemistry B, 106, 12376-12395. doi:10.1021/jp0213506

[33] Nandi, N. and Bagchi, B. (1996) Ultrafast solvation dynamics of an ion in the gamma-cyclodextrin cavity: The role of restricted environment. Journal of Physical Chemistry, 100, 13914-13919. doi:10.1021/jp960134s

[34] Abramczyk, H., Surmacki, J., Brożek-Płuska, B., Morawiec, Z. and Tazbir, M. (2009) The hallmarks of breast cancer by Raman spectroscopy. Journal of Molecular Structure, 924-926, 175-182. doi:10.1016/j.molstruc.2008.12.055

[35] Abramczyk, H., Placek, I., Brożek-Płuska, B., Kurczewski, K., Morawiec, Z. and Tazbir, M. (2008) Human breast tissue cancer diagnosis by Raman spectroscopy. Spectroscopy, 22, 113-121.

[36] Kim, J. and Cremer, P.S. (2001) Investigations of water structure at the solid/liquid interface in the presence of supported lipid bilayers by vibrational sum frequency spectroscopy. Langmuir, 17, 7255-7260.

doi:10.1021/la0017274

[37] Cowan, M.L., Bruner, B.D., Huse, N., Dwyer, J.R., Chugh, B., Nibbering, E.T.J., Elsaesser, T. and Miller, R.J.D. (2005) Ultrafast memory loss and energy redistribution in the hydrogen bond network of liquid $\mathrm{H}_{2} \mathrm{O} . \mathrm{Na}$ ture, 434, 199-202. doi:10.1038/nature03383

[38] Steinel, T., Asbury, J.B., Zheng, J. and Fayer, M.D. (2004) Watching hydrogen bonds break: A transient absorption study of water. Journal of Physical Chemistry A, 108, 10957-10964. doi:10.1021/jp046711r

[39] Ghosh, A., Smits, M., Bredenbeck, J. and Bonn, M. (2007) Membrane-bound water in energetically decoupled from nearby bulk water: An ultrafast surface-specific investigation. Journal of the American Chemical Society, 129, 9608-9609. doi:10.1021/ja073130h

[40] Dwyer, J.R., Szyc, L., Nibbering, E.T.J. and Elsaesser, T. (2008) Ultrafast vibrational dynamics of adeninethymine base pairs in DNA oligomers. Journal of Physical Chemistry B, 112, 11194-11197. doi:10.1021/jp8054119

[41] Elsaesser, T. (2009) Two-dimensional infrared spectroscopy of intermolecular hydrogen bonds in the condensed phase. Accounts of Chemical Research, 42, 1220-1228. doi:10.1021/ar900006u

[42] Abramczyk, H., Brożek-Płuska, B., Surmacki, J. Jabłońska, J. and Kordek, R. (2010) Journal of Molecular Liquids, unpublished.

[43] Elsaesser, T. and Bakker, H.J. (2002) Ultrafast hydrogen bonding dynamics and proton transfer processes in the condensed phase. Kluwer Academic Publishers, Dordrecht.

[44] Abramczyk, H. (1990) IR [nu] s (XH) Absorption band shape in H-bonded complex I: Theory. Chemical Physics, 144, 305-318. doi:10.1016/0301-0104(90)80096-G

[45] Abramczyk, H. (1990) IR [nu] s (XI-I) Absorption band shape of H-bonded complex in condensed media. II: Numerical calculations of the profiles. Chemical Physics, 144, 319-326. doi:10.1016/0301-0104(90)80097-H

[46] Martí, J., Guàrdia, E. and Padró, J.A. (1994) Dielectric properties and infrared spectra of liquid water: Influence of the dynamic cross correlations. Journal of Physical Chemistry, 101, 10883-10891. doi:10.1063/1.467838

[47] Ahlborn, H., Ji, X., Space, B. and Moore, P.B. (1999) A combined instantaneous normal mode and time correlation function description of the infrared vibrational spectrum of ambient water. Journal of Physical Chemistry, 111, 10622-10632. doi:10.1063/1.480415

[48] Ahlborn, H., Space, B. and Moore, P.B. (2000) The effect of isotopic substitution and detailed balance on the infrared spectroscopy of water: A combined time correlation function and instantaneous normal mode analysis. Journal of Chemical Physics, 112, 8083-8088. doi: $10.1063 / 1.481408$

[49] Bansil, R., Berger, R.T., Toukan, K., Ricci, M.A. and Chen, S.H. (1986) A molecular dynamics study of the $\mathrm{OH}$ stretching vibrational spectrum of liquid water. Chemical Physics Letters, 132, 165-172. doi:10.1016/0009-2614(86)80101-4

[50] Silvestrelli, P.L., Bernasconi, M. and Parrinello, M. (1997) $A b$ initio infrared spectrum of liquid water. 
Chemical Physics Letters, 277, 478-482. doi:10.1016/S0009-2614(97)00930-5

[51] Buch, V. (2005) Molecular structure and OH-stretch spectra of a liquid water surface. Journal of Physical Chemistry B, 109, 17771-17774. doi:10.1021/jp052819a

[52] Buch, V., Tarbuck, T, Richmond, G.L., Groenzin, H., Li, I. and Schultz, M.J. (2007) Sum frequency generation surface spectra of ice, water, and acid solution investigated by an exciton model. Journal of. Chemical Physics, 127, Article ID: 204710. doi:10.1063/1.2790437

[53] Torii, H. (2006) Time-domain calculations of the polarized Raman spectra, the transient infrared absorption anisotropy, and the extent of delocalization of the $\mathrm{OH}$ stretching mode of liquid water. Journal of Physical Chemistry A, 110, 9469-9477. doi:10.1021/jp062033s

[54] Buch, V., Bauerecker, S., Devlin, J.P., Buck, U. and Kazimirski, J.K. (2004) Water clusters in the size range of tens-thousands of molecules: A combined computational/spectroscopic outlook. International Reviews in Physical Chemistry, 23, 375-433. doi:10.1080/01442350412331316124

[55] Auer, B. and Skinner, J.L. (2007) Dynamical effects in line shapes for coupled chromophores: Time-averaging approximation. Journal of Chemical Physics, 127, Article ID: 104105 .

[56] Jansen, T.I.C., Zhuang, W. and Mukamel, S. (2004) Stochastic Liouville equation simulation of multidimensional vibrational line shapes of trialanine. Journal of Chemical Physics, 121, 10577-10598. doi:10.1063/1.1807824

[57] Jansen, T.I.C., Dijkstra, A.G., Watson, T.M., Hirst, J.D. and Knoester, J. (2006) Modeling the amide I bands of small peptides. Journal of Chemical Physics, 125, Article ID: 044312

[58] Jansen, T.I.C. and Knoester, J. (2006) Nonadiabatic effects in the two-dimensional infrared spectra of peptides: application to alanine dipeptide. Journal of Physical Chemistry B, 110, 22910-22916. doi:10.1021/ip064795t

[59] Mukamel, S. and Abramavicius, D. (2004) Many-body approaches for simulating coherent nonlinear spectroscopies of electronic and vibrational excitons. Chemical Reviews, 104, 2073-2098. doi:10.1021/cr020681b

[60] Zhuang, W., Abramavicius, D., Hayashi, T. and Mukamel, S. (2006) Simulation protocols for coherent femtosecond vibrational spectra of peptides. Journal of Physical Chemistry B, 110, 3362-3374. doi:10.1021/jp055813u

[61] Choi, J., Hahn, S. and Cho, M. (2005) Amide I IR, VCD, and 2D IR spectra of isotope-labeled $\alpha$-helix in liquid water: Numerical simulation studies. International Journal of Quantum Chemistry, 104, 616-634. doi:10.1002/qua.20543

[62] Everitt, K.F., Lawrence, C.P. and Skinner, J.L. (2004) Density-dependent isotropic Raman line shapes in compressed room-temperature nitrogen. Journal of Physical Chemistry B, 108, 10440-10444. doi:10.1021/jp0379446

[63] Hahn, S., Ham, S. and Cho, M. (2005) Simulation studies of amide I IR absorption and two-dimensional IR spectra of $\beta$ hairpins in liquid water. Journal of Physical Chemistry $B$, 109, 11789-11801. doi:10.1021/jp050450j

[64] Choi, J.H., Lee, H., Lee, K.K., Hahn, S. and Cho, M.
(2007) Computational spectroscopy of ubiquitin: Comparison between theory and experiments. Journal of Chemical Physics, 126, Article ID: 045102. doi:10.1063/1.2424711

[65] Gorbunov, R.D., Nguyen, P.H., Kobus, M. and Stock, G. (2007) Quantum-classical description of the amide I vibrational spectrum of trialanine. Journal of Chemical Physics, 126, Article ID: 054509. doi:10.1063/1.2424711

[66] Jansen, T.I.C., Cringus, D. and Pshenichnikov, M.S (2009) Dissimilar dynamics of coupled water vibrations. Journal of Physical Chemistry A, 113, 6260-6265. doi:10.1021/ip900480r

[67] Berendsen, H.J.C., Grigera, J.R. and Straatsma, T.P. (1987) The missing term in effective pair potentials. Journal of Physical Chemistry, 91, 6269-6271. doi:10.1021/j100308a038

[68] McQuarrie, D.A. (1976) Statistical Mechanics. Harper \& Row, New York.

[69] Frisch, M.J., Trucks, G.W., Schlegel, H.B. and Scuseria, G.E. (2003) GAUSSIAN 03. Gaussian Inc., Pittsburgh PA.

[70] Colbert, D. and Miller, W.H. (1992) A novel discrete variable representation for quantum mechanical reactive scattering via the $S$-matrix Kohn method. Journal of Chemical Physics, 96, 1982-1991.doi:10.1063/1.462100

[71] Morita, A. and Hynes, J.T. (2000) A theoretical analysis of the sum frequency generation spectrum of the water surface. Chemical Physics, 258, 371-390. doi:10.1063/1.462100

[72] Scherer, J.G. and Snyder, R.G. (1977) Raman intensities of single crystal ice $\mathrm{I}_{\mathrm{h}}$. Journal of Chemical Physics, 67, 4794-4811. doi:10.1063/1.434683

[73] Bertie, J.E., Francis, B.F. and Scherer, J.R. (1980) On the determination from Raman spectra of the polarizability derivative with respect to the O-D bond stretching coordinate in the ices. Journal of Chemical Physics, 73, 6352-6353. doi:10.1063/1.440103

[74] Wilson, E.B., Decius, J.C. and Cross, P.C. (1955) Molecular Vibrations. Dover, New York.

[75] Sadlej, J., Buch, V., Kazimirski, J.K. and Buck, U. (1999) Theoretical study of structure and spectra of cage clusters $\left(\mathrm{H}_{2} \mathrm{O}\right)_{n}, n=7-10$. Journal of Physical Chemistry A, 103 4933-4947. doi:10.1021/jp990546b

[76] Adams, D.J. and Dubey, G.S. (1987) Taming the Edwald sum in the computer simulation of charged system. Journal of Computation Physics, 72, 156-176. doi:10.1021/j100169a019

[77] Abramczyk, H. (1991) Absorption spectrum of the solvated electron. I: Theory. Journal of Physical Chemistry, 95, 6149-6155. doi:10.1021/j100169a019

[78] Abramczyk, H. and Kroh, J. (1992) Near-IR absorption spectrum of the solvated electron in alcohols, deuterated water, and deuterated glasses: Lack of observance of the near-IR spectrum in water glasses. Journal of Physical Chemistry, 96, 3653-3658. doi:10.1021/j100188a019

[79] Abramczyk, H. (2004) Femtosecond primary events in bacteriorhodopsin and its retinal modified analogs: Revision of commonly accepted interpretation of electronic spectra of transient intermediates in bacteriorhodpsin photocycle. Journal of Chemical Physics, 120, 1112011132. doi:10.1063/1.1737731

[80] Terentis, A., Uji, L., Abramczyk, H. and Atkinson, G.H. 
(2005) Primary events in the bacteriorhodopsin photocycle: Torsional vibrational dephasing in the first excited electronic state. Chemical Physics, 313, 51-62. doi:10.1016/j.chemphys.2004.12.012

[81] Volkov, V.V., Palmer, D.J. and Righini, R. (2007) Heterogeneity of water at the phospholipid membrane interface. Journal of Physical Chemistry B, 111, 1377-1383. doi:10.1021/ip065886t

[82] Kim, J. and Cremer, P.S. (2000) IR-Visible SFG investigations of interfacial water structure upon polyelectrolyte adsorption at the solid/liquid interface. Journal of the American Chemical Society, 122, 12371-12372. doi:10.1021/ja003215h

[83] Eisenberg, D. and Kauzmann, W. (1969) The structure and properties of water. Oxford University Press, New York.

[84] Yalamanchili, M.R., Atia, A.A. and Miller, J.D. (1996) Analysis of interfacial water at a hydrophilic silicon surface by in-situ FTIR/Internal reflection spectroscopy. Langmuir, 12, 4176-4184. doi:10.1021/la950340b

[85] Torre, R., Bartolini, P. and Righini, R. (2004) Structural relaxation in supercooled water by time-resolved spectroscopy. Nature, 428, 296-299. doi:10.1038/nature02409

[86] Moller, K.B., Rey, R. and Hynes, J.T. (2004) Hydrogen bond dynamics in water and ultrafast infrared spectroscopy: A theoretical study. Journal of Physical Chemistry $A$, 108, 1275-1289. doi:10.1021/jp035935r

[87] Ishiyama, T. and Morita, A. (2009) Vibrational spectroscopic response of intermolecular orientational correlation at the water surface. Journal of Physical Chemistry $C, 113,16299-16302$.doi:10.1021/jp9060957

[88] Nagata, Y. and Mukamel, S. (2010) Vibrational sum- frequency generation spectroscopy at the water/lipid interface: Molecular dynamics simulation study. Journal of the American Chemical Society, 132, 6434-6442. doi:10.1021/ja100508n

[89] Bertie, J.E., Ahmed, M.K. and Eysel, H.H. (1989) Infrared intensities of liquids. 5. Optical and dielectric constants, integrated intensities, and dipole moment derivatives of water and water-d2 at 22.degree.C. Journal of Physical Chemistry, 93, 2210-2218. doi:10.1021/j100343a008

[90] Brubach, J.B., Mermet, A., Filabozzi, A., Gerschel, A. and Roy, P. (2005) Signatures of the hydrogen bonding in the infrared bands of water. Journal of Chemical Physics, 122, Article ID: 184509-1-184509-7. doi:10.1063/1.1894929

[91] Bertie, J.E. and Lan, Z. (1996) Infrared intensities of liquids $\mathrm{XX}$ : the intensity of the $\mathrm{OH}$ stretching band of liquid water revisited, and the best current values of the optical constants of $\mathrm{H} 2 \mathrm{O}(\mathrm{l})$ at 25 degree.C between 15,000 and $1 \mathrm{~cm}^{-1}$. Applied Spectrosctroscopy, 50, 1047-1057. doi:10.1366/0003702963905385

[92] Aroca, R. and Rodriguez-Lorente, S. (1997) Surface enhanced vibrational spectroscopy. Journal of Molecular Structure, 408-409, 17-22. doi:10.1016/S0022-2860(96)09489-6

[93] Walrafen, G.E. (1967) Raman spectral studies of the effects of temperature on water structure. Journal of Chemical Physics, 47, 114-126. doi:10.1063/1.1711834

[94] Walrafen, G.E. (2005) New Raman method for aqueous solutions: $\boldsymbol{\xi}$-function dispersion evidence for strong $\mathrm{F}^{-}$-water $\mathrm{H}$-bonds in aqueous $\mathrm{CsF}$ and $\mathrm{KF}$ solutions. Journal of Chemical Physics, 122, Article ID: 074506. 